\title{
Enhancing the popular music ensemble workshop and maximising student potential through the integration of creativity
}

\begin{abstract}
Ensemble work is a key part of any performance-based popular music course and involves students replicating existing music or playing 'covers'. The creative process in popular music is a collaborative one and the ensemble workshop can be utilised to facilitate active learning and develop musical creativity within a group setting. This is a report on a reflection into the practice of the ensemble workshops within the author's own further education context. It is proposed that in order to unlock creative potential, develop active group learning and add value to the student, a new approach to pedagogy within ensemble work is required. This approach involves scaffolding learning through the setting of collaborative, creative tasks and provision of a mentoring environment in which students can feel safe to experiment.
\end{abstract}

\section{Keywords}

Collaboration, creativity, ensemble workshop, pedagogy, performance, popular music, transferable skills

\section{Introduction}

In the popular music ensemble workshop, groups or bands of music students perform popular music together and are critiqued and assessed by one or more tutors on the musical, technical and performance merits.

Since early 2007 I have been leading the ensemble workshops as head of musical studies at a private school for popular music based in the UK within the post-16, further education (FE) sector. At the time I took on this role, the ensemble workshops offered predominantly a platform for the replication of existing music. Whilst the skill of listening to, working out and replicating music (also known as playing 'covers') is a useful one, it is only one aspect of musicianship required for popular music. The integration of creativity within the ensemble workshop is important for student engagement and for drawing out the creative and musical potential of the students. With this element of creativity lacking in my context, I found that the ensemble workshops 
were becoming routine and dry, lacking clear purpose or context and failing to further the creative and musical potential within the students.

This article helps to extend the discourse into the ensemble workshop by presenting a practical reflection on the pedagogic approach within my own ensemble curriculum. The article also high- lights important elements for consideration for anyone undertaking a similar such review.

\section{Contextualising the ensemble workshop for popular music}

Popular music is often taught using a similar pedagogic approach to that of classical music (Lebler, 2007). In classical music the focus is on analysis and performance of a musical canon rather than the more informal, creative and improvised practices common to popular music. Furthermore, classical music tends to separate the role of composer and performer, whereas in popular music from the 1960s onwards, this distinction is blurred, as musicians are all expected to have input into the creative process. As Boespflug (2004) identified, the approach to ensemble pedagogy within classical music does not teach or facilitate the types of skills required for popular music.

\section{Creativity}

Creativity is frequently cited by employers as a sought-after quality in graduates (Chan, 2012). The modern world increasingly values creativity in its employees (Simpson, 2009) and education is under increasing pressure to ensure students are in possession of this important attribute. For the purposes of this article the following definition of creativity from Robinson (2001) offers a helpful summary: '[creativity is] the process of having original ideas that have value' (p. 67). Simpson (2009) added further definition to this describing the act of being creative as 'identifying a pattern that can be broken, a new way to use an already existing concept or product or by taking the influence from society and tweaking it so it has a fresh new look' (p .5).

\section{Flow experiences and creativity}

Csikszentmihalyi (2002) suggested that creativity often flourishes when clear boundaries or parameters are set, allowing for students to achieve what he refers to as 'optimal' or 'flow' experiences. Whilst flow theory does not specifically relate to music performance 
it has a high degree of relevance. In this context flow can refer to highly rewarding and motivating experiences achieved when the student is suitably creatively challenged and immersed in the activity of performing and learning. These types of flow experiences tend to be more common when students perform music in which they have been personally involved in the creative processes, as there is a heightened sense of personal ownership and investment.

\section{Barriers to flow within education}

The traditional education environment, however, can prove a barrier to producing an environment where 'flow' experiences can be realised. Wistreich (2012) suggested that the traditional performance assessment methodology within formal institutions can be a source of stress and unhealthy competition amongst students. This gives rise to 'academic "fetishizing" of "accurate" performances at the expense of communication, pleasure and other desirable qualities that normally motivate musicians and their listeners' (p. 7).

\section{Fear of failure}

Fear of failure can lead to a desire to play safe and avoid presenting music that is new and original. Robinson (2009) states that, 'If you are not prepared to be wrong then you will never produce anything original' (p. 74), yet a willingness to be 'wrong' does not fit within the traditional ensemble assessment culture that Wistreich refers to; where rewards are more readily given for accuracy of performance rather than creative and expressive qualities. To be prepared to be wrong requires a learning environment in which there exists a high level of trust, mutual respect and good relation- ship between staff and student.

\section{A creative learning environment}

Rather than an environment where the teacher is seen as master, in possession of the knowledge, and the student as the novice, a mentoring relationship between staff and student provides a learning environment more conducive to creativity where 'the learner is no longer an "object" of pedagogy, but becomes a creator in the field being taught' (Mazzola, Park, \& Thalmann, 2011, p. 161). 


\section{Summary}

The ensemble workshop within popular music requires its own specialist pedagogy that is wholly different from that of classical music and should provide more than just the opportunity for per- forming 'covers'. Whilst there are many benefits to embedding creativity into the ensemble work- shop, careful consideration needs to be given to the learning environment and context within which it exists. The sections that follow detail the process of enhancing the ensemble workshop curriculum and the creation of a suitable learning environment within my own FE institution.

\section{Enhancing the ensemble workshop}

\section{Initial response to the problem}

In seeking a solution to the problem described in the introduction, the initial response was to set students the task of writing and performing original music as part of their workshops. This appeared to be the correct decision as students were being directed towards higher levels of creative engagement. However, the shift from replicating existing music to writing original music from scratch tended to prove a difficult transition for students.

\section{Lack of creative context}

The problem was that creating original music tabula rasa, with no context or brief was too vague and open ended for students to be appropriately challenged and focussed. Those students who did not consider themselves song writers tended to find the prospect of creating something from scratch intimidating, believing they had little to offer to the creative process. Even students who did con- sider themselves songwriters, and thus were not intimidated by the prospect of writing original music, tended to have limited compositional vocabulary and stayed within their own stylistic com- fort zones. After reflecting back on the first year of leading the ensemble workshops, a scaffolding approach to learning seemed the most appropriate direction to go in. This took the form of prescribed, creative tasks (an example of which is given in Figure 1), allowing students to progress gradually into more creative practices and providing a broader compositional experience. These tasks presented real-life scenarios or contexts that require a creative and collaborative response and offered a safe framework for the students to work within, giving a starting point and directive focus for their creative efforts. 


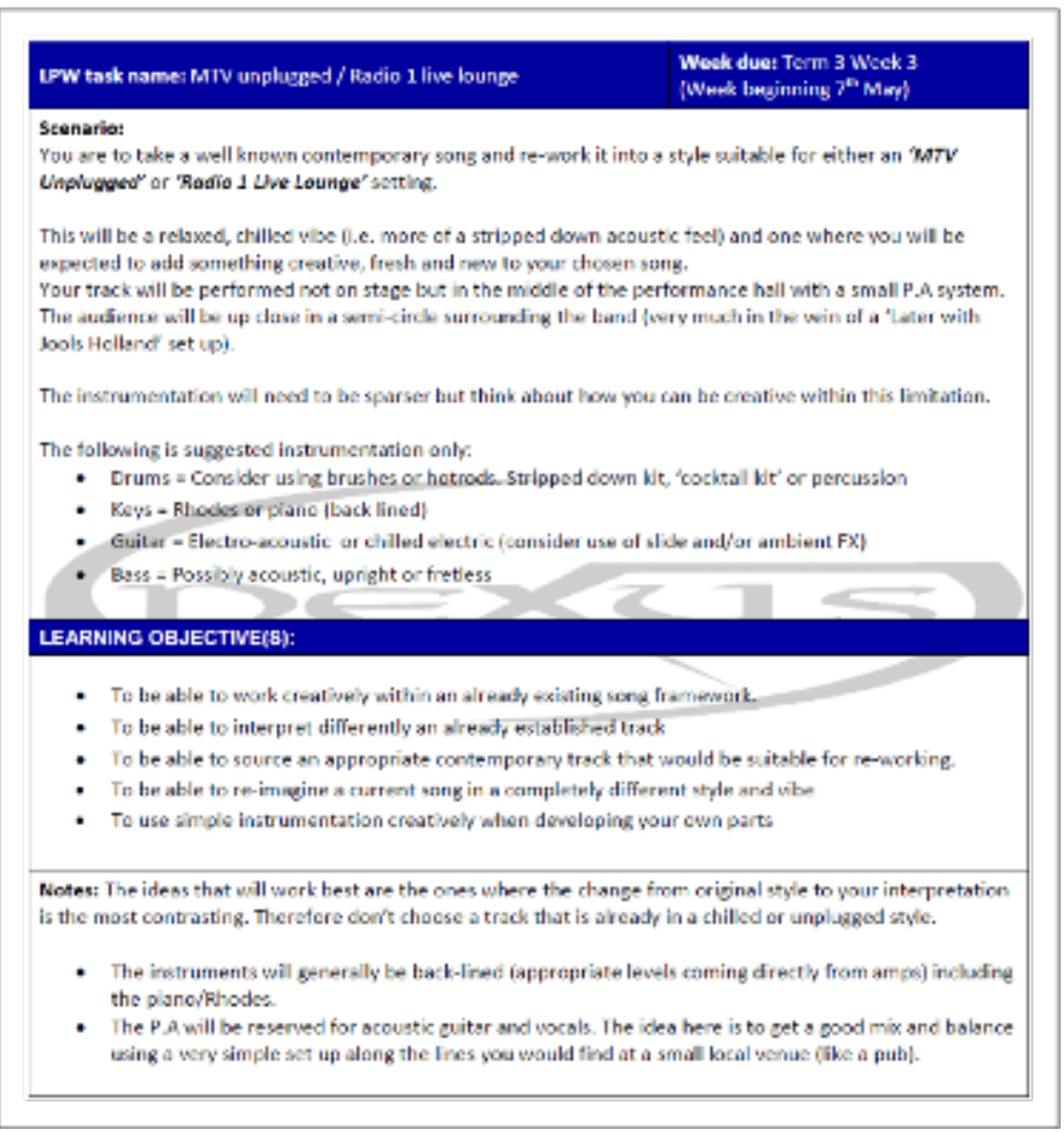

Figure 1. Example of a creative task brief given to students for a popular music ensemble workshop.

\section{Developing the curriculum}

Before developing the creative tasks an examination of the key skills required for popular musicians and the most common, real-life playing scenarios was first undertaken. This was achieved through collective discussion amongst teaching staff as to their experience and understanding of

the popular music industry. Key skill areas identified were:

- developing instrumental/vocal technique;

- $\quad$ imaginative instrumentation and band arrangement; 
- $\quad$ visual presentation (such as dress code, stage arrangement and performance);

- visual communication and band interaction;

- $\quad$ effective use of staging and live performance equipment;

- $\quad$ effective rehearsal strategies;

- $\quad$ professional sound check etiquette (including use of in-ear and fold-back monitors)

- $\quad$ coping with unexpected scenarios and technical issues whilst performing.

\section{Practical coaching within the ensemble workshop}

The skills outlined above represent the main areas of learning and development that students are coached in and assessed upon. It is important to note that, although not all these skills are new requirements within the popular music curriculum in general, it is the practical coaching and development of them within the context of the ensemble workshop that was significant in developing the pedagogic approach in my own working context. Because of this, skills and concepts being taught can be directly linked to, rather than being separate from, a practical performance setting. This is achieved by having a representative tutor from each of the five instrument departments (guitar, bass guitar, keyboards, drums and vocals) present on the ensemble panel. Although it is common practice for specialist instrument coaching and one- to-one lessons to be included in music programmes within the UK, it tends to exist separate from the ensemble workshop context. This means students do not always receive specific feedback and instruction from their respective specialist tutors directly within a performance context.

\section{Contextual relevance}

Having defined the skill areas that were to form the basis of the new curriculum (see the Developing the curriculum section), it was then possible to begin defining the creative tasks. Strong and clear links between the tasks given and the professional scenarios to which they relate is an essential component in the new curriculum. As a form of situated learning, the skills and playing environments identified by teaching staff were contextualised within a series of imaginary scenarios to which students are required to respond. Assignment briefs for each task detailed a scenario or context that related to an aspect of the music industry, allowing for clear understanding as to how the task will 
develop the student as a creative musician. Clear rules and boundaries helped focus the students' creative thinking and provided an environment in which flow experiences can be readily achieved.

\section{The creative task brief: a practical example}

\section{The scenario}

It is common practice within popular music to create alternative, acoustic-instrumentbased ver- sions of musical pieces for radio performances when sound level or space restrictions exist or where an alternative, more accessible version is desired. This real-life scenario forms the basis for one of the creative tasks set in the new curriculum. A taskbriefing document summarising the context, the skills involved and how to approach it were given to the students. Using descriptors that students would almost certainly be familiar with, in this case 'MTV unplugged' and 'Radio 1 live lounge', helped contextualise in the students' minds that which they were being asked to do. They would have been able to bring to mind exemplars, to help them become more comfortable and invested in the task, as it related to their own cultural experience.

\section{Radio 1 live lounge/MTV unplugged task}

In the recorded example (URL given in the Appendix), the key skill areas outlined earlier could be regarded as having been covered within this one task. It is a re-work of the 2005 popular music track 'One Thing' by the artist Amerie. The original track featured a fastpaced and heavily produced sample-based arrangement that did not naturally lend itself to being performed live in a more acoustic band setting. Challenges would have been identifying the key elements of the track and adapting them to work within a more acoustic, stripped back arrangement whilst still creating an interesting and captivating musical performance. For example, students had to think creatively about the arrangement and instrumentation in order to capture the key musical elements of the studio version, but within the narrower confines of an acoustic band set up. This required effective use of musical equipment, exploring and exploiting the range of sounds achievable in order to create dynamic and timbral/textural interest. This can be heard in the drum-kit part, which used a steel brushes technique, pedalled hi-hat and an accenting pattern on the snare drum to capture the complex and layered drum sample and percussion parts on the original version. The electric guitar made use of effects such as digital delay and wah-wah pedal to act as an upper texture; sustaining across the chord progression to help link and smooth the unusual changes (I, III, II, I, IV played as major 
chords). Arriving at the finished performance required the employment of good working and rehearsal strategies, and dialogue within the group had to be both creative and collaborative, making evaluative decisions as well as critical assessment of other band members' ideas. Undertaking this task developed the students' musical and creative abilities in ways that would not have been possible through performing covers.

Figure 1 shows the task briefing given to students. It outlines the performance scenario, gives advice and direction as well as setting boundaries and detailing learning objectives.

\section{Providing a learning environment conducive to creativity}

As identified earlier, the provision of a learning environment in which students can feel safe to experiment was as essential as finding the right approach to pedagogy. Emphasis was placed on mentoring relationships through staff and students working together creatively and engaging in joint activity, an approach that sat well within the more informal learning processes that are characteristic of popular music. This required a flattening of hierarchical structures that can create distance between staff and student.

The trusting relationship between staff and students creates a very safe learning environment in which mistakes, as part of a healthy learning process are allowed and encouraged. (TB: Guitar student - End of year student questionnaire, June 2012)

\section{Assessment}

Students were assessed in the ensemble workshops, and these assessments sat within a context in which guidance and coaching were provided both in rehearsals and performances, helping students to reach their potential, rather than simply assessing an end performance with a mark awarded sometime later.

Individual tutors gave formative feedback to each student during the rehearsal and workshop stages as well as directly after the assessed performances, although this did not count towards the awarded marks. Summative assessment was awarded as a group mark expressed as a percentage and based on a number of performances throughout the programme rather than a single end-of- programme assessed performance. This allowed students the opportunity to implement the forma- tive feedback they receive to help improve marks as they progressed through the programme. Responsibility for assessing the band performances fell to two tutors at a time with input received from other members of the panel. Whilst performances were not open to the general public, the 
students' peer group (around 20-30 students) were in attendance.

Each creative task brief presented the learning objectives for that task and students were assessed according to the extent to which they met these. In addition to these there were the general skill areas (see the Developing the curriculum section) against which students were assessed that con- tribute to a final mark for that task.

\section{Feedback}

A written report was given to each student from their instrument tutor, giving critical evaluation and highlighting strengths and areas for future development. This provision of immediate feedback was of significant value as it allowed improvements to performance skills to be implemented promptly. The active contribution of instrument-specific teaching staff in this way allowed clear instruction and guidance pertinent to the student to be given, rather than more a more generalised feedback approach. This feedback was not limited only to instrumental technique but also covered all the skill areas detailed earlier.

Although it was not impossible for any one of these areas to be assessed by a member of staff experienced in popular music, but not sharing the student's instrument specialism, providing a panel of instrument-specialist staff allowed for a greater degree of specificity and targeting of feed- back as well as increasing contact time for students.

\section{A learning community}

Incorporating instrument teaching staff into the ensemble workshop allowed both student and tutor to engage in the learning process together, bringing professional training directly into the ensemble setting. An effective example of this from within my own FE college environment was our Christmas concert. The Christmas concert, which was previously a recital-based output of the ensemble workshop, has now become a full themed performance with a set, acted story line and music being produced. It enables staff and students to work together creatively in achieving a shared goal and incorporating many varied, multifaceted elements such as stage craft, composition, stage design and planning, set construction, lighting and choreography. For example, in the construction of the set, many staff and students were involved in design work, construction work and set dressing. Solutions to problems, such as enabling the design of the set to reflect the story, as well as working on ways to physically realise creative 
concepts, required a joint effort. How to stage and choreograph musicians during the performance gave the opportunity to involve students in the creative process, helping them to understand the practical realities of presenting a musical performance.

Through this, many valuable lessons and transferrable skills have been delivered in an informal learning environment. Some of the music in the concert involved staff performing together with students and working alongside them in the later stages of the rehearsal process. This proved a significant factor in helping reduce staff/student distance and students valued the experience of rehearsing and performing alongside staff.

Seeing things come to completion will be a lasting memory for me from the Christmas concerts. It was amazing to see everyone work together to create such an amazing concert.

(NJ: Vocal student: End of year student questionnaire, June 2012)

\section{Deep learning}

The benefit of working in this way is that now students tend to give considerable time and effort into the more holistic elements of their ensemble performances rather than only doing what is required to pass. Examples include enhancing the overall performance through use of a particular stage layout, set design (such as banners, specific clothing, use of performance paraphernalia - such as glow sticks and ultraviolet (UV) paint), choosing lighting effects and creation of video media to accompany the musical performance. Often this is undertaken without any prompting from staff, representative of the students' desire to experiment and excel within their ensemble performances, showing a deep learning approach rather than a surface learning approach.

\section{Summary}

I have shown that through a new pedagogic approach to the ensemble workshops at my own FE institution, students have discovered they can produce work exhibiting high levels of musical creativity and quality within the terms defined earlier by Simpson. They have become more musically diverse, adept and able to perform in live contexts outside of the class room setting. This has helped to improve personal confidence and empowerment through a sense of achievement and pride in work produced.

They [ensemble workshops] challenged me and made me realise that I have a lot more ideas in my head musically than I thought there were. 
Furthermore, the nature of a mentoring learning environment enables the forming of strong, rich relationships through shared experience and creative collaboration, as well as opportunities for shared experience between staff and student outside of the classroom setting.

$$
\begin{aligned}
& \text { Working with a band has really helped prepare for working with future } \\
& \text { colleagues. Being able to share ideas, make decisions and work as a } \\
& \text { team are key skills for any job. } \\
& \text { (GB: Vocal student - End of year student questionnaire, June 2012) }
\end{aligned}
$$

\section{Discussion}

I have argued that the ensemble workshop for popular music requires its own pedagogy that is distinct from that of classical music, offering more than just opportunities for musical replication. The ensemble workshop experience can be enhanced through the integration of creativity into the curriculum through development of creative tasks situated and contextualised within professional performance scenarios. This has enhanced the student experience, enabling them to make a more gradual and effective progression from replication to the creation of original music.

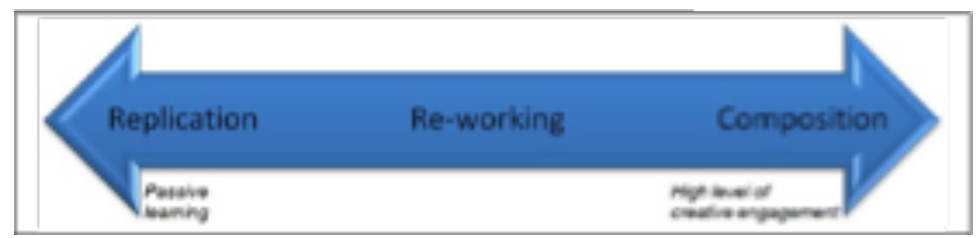

Figure 2. Representation of the continuum of creative engagement in ensemble workshops.

\section{The continuum of creativity}

When considering the progression from replication to the creation of original music I have found it helpful to think of creativity within ensemble workshops as existing on a continuum on which varying levels of engagement in the creative process can be said to exist (see Figure 2).

I consider that there are two opposite poles on the continuum. Replication represents 
matching a previously composed piece of music as closely as possible without contributing anything new or deviating from the original. By doing this, the individual's musical and creative contribution is at a low level and this could encourage a passive learning approach over time. At the other end of the continuum is composition. This refers to the creation of original music that differs from previously composed music, within the broad stylistic norm of popular music, enough to be considered as a new and separate musical product.

In the middle of the continuum I have situated the concept of re-working. I choose to define re- working as the process that utilises existing music as stimuli, or frameworks, with which to com- bine creative ideas to produce new and valuable music. I have found that this midway point provides a helpful stepping stone towards the objective of being able to produce diverse and creative original music. This has produced a far deeper approach to learning than with the previous pedagogic approach and has helped to creatively engage all students.

\section{The learning environment}

A necessary catalyst to creativity is the provision of a learning environment where effective teacher and student interaction improves the quality of work and adds value to the student learning experience. The dynamic of staff and student collaboration need not necessitate significant further investments of contact time. It can be a shift in pedagogical approach or else small investments of contact time given to collaborative working opportunities that enrich the student learning experience. Team building events and opportunities for staff and students to work together creatively are good ways of encouraging and building a positive learning community.

\section{Conclusion}

There has been significant benefit in reflecting on the approach to pedagogy for the ensemble workshop and asking whether enough is being done to provide students with the right framework and opportunity to fulfil their creative potential. If significant and creative new music is not being produced as a direct result of the ensemble workshop then it is worth questioning what is being produced and what benefit it is providing to the student.

In the introduction, it was established that the skills one learns through a replication or 
'covers' approach alone, whilst important, do not completely reflect the requisite skills for a popular music context. A key goal of any popular music pedagogy, therefore, should be to enable students to become active participants and creative contributors to the field. With respect to the ensemble workshop, if replication is the pedagogic hegemony students may be failing to develop the creative and divergent thinking skills that are important for many of the employment opportunities in the popular music industry.

Clearly a student well developed in the creative application of their musical skills will have a competitive advantage over a similar such student that had not developed those same skills. For the popular music ensemble workshop to be effective in training musicians in the kinds of skills required by a music industry reliant on creativity, a new pedagogic approach must be developed that is fit for purpose and relevant specifically to popular music.

\section{References}

Boespflug, G. (2004). The pop music ensemble in music education. In C. X. Rodriguez (Ed.), Bridging the gap: Popular music and music education (pp. 191-203). Reston, VA: MENC.

Chan, K., Leung, V., Tsang, L., and Yip, T. (2012) 'An Exploratory Study of Consumers,' Attitudes Toward Advertising of Legal Professionals in Hong Kong. Asian Journal of Business Research 2 (2), 70-86

Csikszentmihalyi, M. (2002). Flow: The classic work on how to achieve happiness. London: Rider. Lebler, D. (2007). Student-as-master? Reflections on a learning innovation in popular music pedagogy.

International Journal of Music Education, 25, 205-221. Mazzola, G., Park, J., \& Thalmann, F. (2011). Musical creativity: Strategies and tools in composition and improvisation. Berlin/Heidelberg, Germany: Springer. Robinson, K. (2001). Out of our minds: Learning to be creative. London, UK: Capstone. Robinson, K. (2009). The element. London, UK: Allen Lane. Simpson, M. (2009). The importance of creativity on our global society and in today's educational system.

Waco, TX: Baylor University. Wistriech, R. (2012). Teaching and assessing collective performance skills in a university music department.

Retrieved from http://www.cetl4musicne.ac.uk/resourcesarchive5.html 


\section{Appendix}

Recorded video example of student work: re-work of the song 'one thing' by Amerie:

http://www. youtube.com/watch?v=iT-N-

66mQcQ\&feature $=$ share \&list $=$ UUZCiOlFPOyq-sDiLjItd9DA

The original track can be heard as a comparison at:

https://www.youtube.com/watch?v=bbqVg_23otg\&feature=youtu.be 